\title{
Numerical Evaluation of the Influence of Fuel Generation on the Geometry of a Diffusion Flame: Implications to Micro-Gravity Fire Safety
}

\author{
Sebastien Rouvreau, Pierre Cordeiro, Pierre Joulain and Hui Ying Wang \\ Laboratoire de Combustion et de Detonique, ENSMA \\ 1 av Clement ADER, B.P. 40109 \\ 86961 FUTUROSCOPE-Chasseneuil du Poitou CEDEX \\ FRANCE
}

and

Jose L. Torero

School of Civil and Environmental Engineering

The University of Edinburgh

Edinburgh, EH9 3JN

United Kingdom

\begin{abstract}
S
Improvement in the understanding of the structure of flames in micro-gravity is a critical issue for spacecraft fire safety. A numerical study of the influence of a gas diffusion flame on a flow of air over a flat plate in micro-gravity environment is presented in this paper. 3D DNS simulations with a mixture fraction model for combustion have been performed with an adaptation of the Fire Dynamics Simulator code (FDS) developed at NIST. A particular flow speed representative of ventilation in spacecrafts and a fuel injection speed comparable to those induced by pyrolysis is used to illustrate the effect of the sample geometry, the fuel injection and thermal expansion of the flow characteristics. This work is benchmarked with experiments conducted on board of the CNES Airbus-300 and with the numerous observations available in the literature. It was observed that a leading edge can cause velocity overshoots and that fuel injection can have a significant effect in propagating the pressure perturbations downstream. Nevertheless, the calculations show that thermal expansion affects the flow in a much more dramatic way, thus becomes the most important parameter to be considered when evaluating the precision of analytical models.
\end{abstract}

KEY WORDS: MICROGRAVITY, DIFFUSION FLAME, SIMULATION

\section{INTRODUCTION}

The investigation of low Reynolds number flows over solid surfaces has been the subject of many studies [1]. Most of the work relates to the multiple applications in the areas of lubrication and heat transfer and almost no attention has been given to highly exothermic reacting low Reynolds number flows. The main reason being that highly exothermic reactions, such as those common in combustion processes, result in buoyantly induced flows that are generally characterised by Reynolds numbers greater than 1000 . The current status of fire safety practices in different existing and planned space facilities has 
been addressed by many authors [2-7] and recent related progress has been summarised in references $[8,9]$. These studies show a need for fundamental combustion studies in micro-gravity to properly establish fire safety protocols in environments that become every day more challenging. A common fire scenario is that of diffusion flame established over the surface of a condensed material. In normal gravity, temperature gradients result in natural convective flows that are laminar when scale is small and leading to transition to turbulence as the size of the fuel increases. In spacecrafts, where buoyancy is negligible, the flow is limited to that induced by the ventilation system. Characteristic HVAC velocities are of the order of $0.1 \mathrm{~m} / \mathrm{s}$, therefore the flow is expected to be laminar and to some extent parallel to the surfaces. The complex mixed flow (often turbulent) fire scenario observed in normal gravity is reduced to the classical combustion problem first described by Emmons [10]. The problem of a chemically reacting boundary layer flow over a flat plate, as described by Emmons, is that of the incompressible boundary layer flow with blowing. The assumptions correspond to a classical Shvab-Zeldovich approach where gravity is neglected. This geometry has been considered as being one of great relevance to fire safety for spacecraft [11] and the Emmons [10] formulation has been proposed as a candidate to interpret current material flammability procedures [12]. Nevertheless, debate over the limitations of this theoretical formulation is still current. This debate is further supported by a number of experiments conducted using porous gas burners that have showed the potential of flame-induced flow perturbations that could limit the use of the Emmons formulation. The flow perturbations described mostly relate to velocity overshoots close to the reacting zone and their effect on flame stability. The first to observe velocity overshoots near the reaction zone were Hirano and co-workers [13,14]. Ramachandra and Raghunandan [15], studied the effect of injection and free stream velocities on the stability of vaporised $\mathrm{n}$-heptane flames and provided similar explanations to those proposed by Hirano and co-workers [13,14]. Lavid and Berlad [16], in their theoretical study, attributed the pressure overshoots to buoyancy. Andreussi and co-workers [17-19] incorporated variable properties to a theoretical analysis very similar to that of Emmons [17-18] and compared a numerical solution to experiments conducted with ethyl-alcohol [17-19], concluding that the Shvab-Zeldovich formulation is only adequate for free stream velocities higher than $1.2 \mathrm{~m} / \mathrm{s}$. Nakagawa et al. [20] tried to establish that an improper definition of the gas phase properties is the cause of the velocity overshoot whereas no velocity distributions were presented in their work. A different approach was followed by Ha et al. [21]. A study of the leading edge was conducted in an attempt to provide a conciliating explanation to the velocity overshoots. Cold and reacting flow velocity measurements and flow visualisation together with elliptic numerical calculations were used to describe the separation close to the leading edge. The above studies show clearly that flow perturbations exist, nevertheless a complete explanation has not been attained, and furthermore the impact of these perturbations on the potential use of Emmons theory for the description of low Reynolds number diffusion flames still remains unclear.Torero et al. [22] showed experimentally that with a gas burner and for velocities relevant to spacecraft and in micro-gravity, that these flow perturbations could lead to separation of the flow close to the leading edge of the flame. Emmons demonstrated theoretically that flow separation makes unusable boundary layer theory since it precludes convective heat transfer from the flame towards the fuel surface, thus eliminating the fuel source. An early attempt to model these experiments numerically did not show separation of the flow [23] but the discrepancy was attributed to the assumption that the flow was of parabolic nature. By conducting experiments with PMMA in micro-gravity, Vietoris et al. $[11,24]$ showed that flow separation occurred for 
free stream velocities greater than $0.1 \mathrm{~m} / \mathrm{s}$ and was induced by an enhancement of radiative feedback generated by the glowing of soot. For lower velocities, the selfregulating nature of fuel pyrolysis serves to retain the original flow assumptions proposed by Emmons and therefore makes possible the use of this theoretical development to describe micro-gravity fires. Although it has been shown that the fundamental principles used by Emmons [10] to describe the nature of laminar diffusion flames remains valid and that it can be used to enhance current fire safety practices for micro-gravity environments. It is clear that the simplification of a free stream velocity used to achieve the analytical solution might result in significant error in the prediction of flame lengths and stand-off distances. Analytical solutions seem therefore limited. The current work aims to use a numerical tool [25] to study in detail the flow perturbations induced by a diffusion flame at flow conditions representative of spacecraft heat and ventilation systems. Many of the perturbations on the flow induced by the fuel and flame are truly 3-D. Thus the use of a $2 \mathrm{D}$ calculation, even if it enables for a much more refined grid, is precluded. The numerical results will be benchmarked with gas burner experiments similar to those presented by Torero et al. [22]. It was considered more appropriate for the purposes of this study to use gas burner experiments because it minimizes uncertainty in the definition of the fuel surface boundary condition.

\section{CALCULATION TOOL AND COMPUTATIONAL DOMAIN}

The numerical tool used for the simulations conducted throughout this study have been made using the Fire Dynamics Simulator (FDS) code developed at NIST by McGrattan et al. [25]. This code has been developed to allow the modelling of many different and complex fire scenarios with a reduced calculation time. For this purpose FDS uses an approximated expression of the Navier-Stokes equations where acoustic waves are filtered whereas it still permits big density and temperature changes. The characteristic velocities corresponding to this study preclude the use of the filter therefore FDS has been used in its Direct Numerical Simulation (DNS) version. Although the combustion chemistry is expected to be complex for the present scenario, it was considered that for a benchmark study that is to be compared to an analytical solution, a simple mixture fraction combustion model was appropriate. The hydrodynamic model is based on a classical reactive flow model:

Mass conservation

$$
\frac{\partial \rho}{\partial t}+\vec{\nabla} \cdot \rho \vec{u}=0
$$

Species conservation

$$
\frac{\partial}{\partial t}\left(\rho Y_{i}\right)+\vec{\nabla} \cdot \rho Y_{i} \vec{u}=\vec{\nabla} \cdot(\rho D)_{i} \vec{\nabla} Y_{i}+\dot{W}_{i}^{\prime \prime \prime}
$$

Momentum conservation

$$
\rho\left(\frac{\partial \vec{u}}{\partial t}+(\vec{u} \cdot \vec{\nabla}) \vec{u}\right)+\vec{\nabla} p=\rho \vec{g}+\vec{\nabla} \cdot \bar{\tau}
$$

Energy conservation

$$
\frac{\partial}{\partial t}(\rho h)+\vec{\nabla} \cdot \rho h \vec{u}-\frac{D p}{D t}=\dot{q}^{\prime \prime}+\vec{\nabla} \cdot k \vec{\nabla} T+\vec{\nabla} \cdot \sum_{i} h_{i}(\rho D)_{i} \vec{\nabla} Y_{i}
$$

The filter is then applied to Navier-Stokes equations and the energy source term is included in the expression of velocity divergence such that energy equation is not solved explicitly [25]. The combustion model is based on the assumption that combustion is 
mixing-controlled. Thus every species involved can be described in term of the mixture fraction $\mathrm{Z}(\mathrm{x}, \mathrm{t})$, a conserved quantity that gives a normalised mass ratio between fuel and oxidiser. Taking the most general way to express a chemical reaction $v_{O}[O]+v_{F}[F] \rightarrow \sum_{p} v_{p}[p]$ where $v_{\mathrm{i}}$ are stoechiometric coefficients for the overall combustion process involving fuel $\mathrm{F}$ and oxidiser $\mathrm{O}$ and giving products $\mathrm{P}$, leads to a relation between mass consumption of fuel and oxidiser:

$$
\frac{m_{F}^{\prime \prime \prime}}{v_{F} M_{F}}=\frac{m_{O}{ }^{\prime \prime}}{v_{O} M_{O}}
$$

Then the mixture fraction can be defined as :

$$
Z=\frac{s Y_{F}-\left(Y_{O}-Y_{O}^{\infty}\right)}{s Y_{F}^{\infty}+Y_{O}^{\infty}} \quad ; \quad s=\frac{v_{O} M_{O}}{v_{F} M_{F}}
$$

where $\mathrm{M}_{\mathrm{O}}$ and $\mathrm{M}_{\mathrm{F}}$ are molecular masses of oxygen and fuel respectively. $\mathrm{Z}$ satisfies the relation

$$
\rho \frac{D Z}{D t}=\vec{\nabla} \cdot \rho D \vec{\nabla} Z
$$

which is obtained from a linear combination of the conservation equation for fuel and oxidiser. The assumption of infinitely fast chemical reaction rate leads to the following definition of the flame

$$
Z(\vec{x}, t)=Z_{f} \quad \text { where } \quad Z_{f}=\frac{Y_{O}^{\infty}}{s Y_{F}^{\infty}+Y_{O}^{\infty}}
$$

The fact that this assumption forbids coexistence of fuel and oxygen leads to the following state relation:

$$
Y_{O}(Z)= \begin{cases}Y_{O}^{\infty}\left(1-Z / Z_{f}\right) & Z<Z_{f} \\ 0 & Z>Z_{f}\end{cases}
$$

Once this relation has been established, the local heat release rate can be obtain by using the relation between mass consumption of oxygen and local heat release rate first defined by Huggett [26]:

$$
\dot{q}^{\prime \prime}=\Delta H_{O} \dot{m}_{O}^{\prime \prime \prime}
$$

where $\Delta H_{O}$ is the heat released per unit of mass of oxygen consumed [26] and where the mass consumption of oxygen is obtain from the following expression :

$$
-\dot{m}_{O}^{\prime \prime}=\vec{\nabla} \cdot\left(\rho D \frac{d Y_{O}}{d Z} \vec{\nabla} Z\right)-\frac{d Y_{O}}{d Z} \vec{\nabla} \cdot \rho D \vec{\nabla} Z
$$

A few adjustments to the code have been made by the authors and involve mainly an adaptation of the mixture fraction model to allow its use in a DNS calculation. The mixture fraction model already existed in FDS but its use was originally restricted to LES calculations. The calculation domain is $300 * 200 * 150 \mathrm{~mm}$ in the X Y and Z directions respectively (Figure 1). The regular eulerian grid contains $150 * 100 * 75$ grid cells, which gives a cell size of $2 \mathrm{~mm}$ in each direction. This cell size has been chosen after testing that results are insensitive to a further refinement of the mesh $(1 \mathrm{~mm}$; $0.5 \mathrm{~mm}$ ). Even further refinement would certainly give a better prediction in sensitive areas like at the leading edge of the burner but nevertheless, most of the phenomena of interest are of much larger scale, thus the actual grid is appropriate. A flow of air with a top hat velocity profile is imposed on the $\mathrm{X}=0$ plane which will be referred as the 
entrance of the domain in this paper. The porous burner is $60 * 60 \mathrm{~mm}$ and is embedded in the $\mathrm{Z}=0$ plane, centred in the $\mathrm{Y}$ direction and at $40 \mathrm{~mm}$ from the entrance of the domain. The boundary condition for the four other sides of the domain, e.g. $x=X_{\max }, y=0$, $\mathrm{y}=\mathrm{Y}_{\max }$ and $\mathrm{z}=\mathrm{Z}_{\max }$, is that of a passive opening: ambient temperature, ambient pressure and zero velocity gradients. This set up has been chosen in order to stick as much as possible to experimental condition used by Torero et al [22] and Vietoris et al [11, 24].

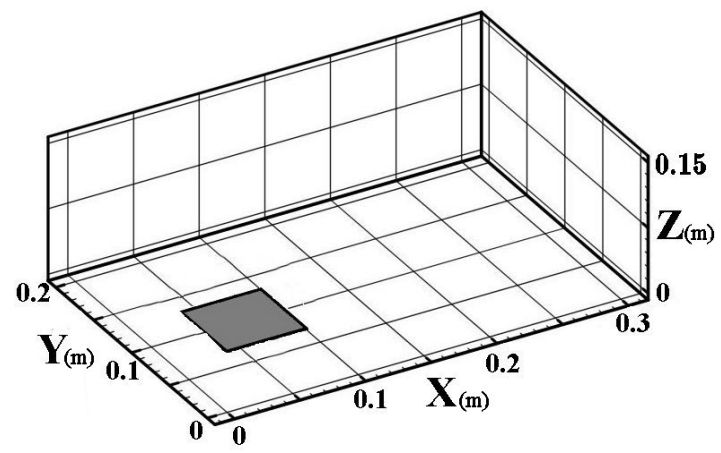

Figure 1: $\quad$ Computational domain. The air flow is introduced through the $X=0$ plane and the fuel is injected perpendicular to the $Z=0$ plane and through the grey area. The computational domain mimics the experimental geometry presented in references [11,22 and 24].

\section{RESULTS}

The studies summarized in the introduction [10-24] show that three different parameters seem to affect the flow structure, the geometry of the plate leading edge, the fuel injection and thermal expansion induced by combustion. These three parameters will be studied systematically and the results described independently in the following sections. The geometry of the leading edge was chosen to conform to the experimental conditions and to minimize its impact on flow separation [11,21] therefore in this study a single geometrical configuration will be used. The first part of this study explores the effect of the presence of the plate on the characteristics of the flow. The free flow over a flat plate with no fuel injection is characterized and the results are compared with observations previously reported in the literature. Particular emphasis is given to the pressure perturbation at the leading edge of the flat plate first described by Hirano [13,14]. Fuel injection is then introduced but the chemical reaction is not allowed to proceed to prevent thermal expansion. The influence of fuel injection is then studied systematically. Finally, a comparison between the reacting flow and the isothermal flow serves to provide some insight on the influence of energy release on the flow and on the geometry of the flames.

\section{Flow Over a Flat Plate}

In order to quantify correctly the influence of fuel injection speed and energy release on the principal flow it is first needed to have a careful look at the isothermal flow without injection. A single flow condition will be presented as an illustrative example of the effect that the plate has on the flow. The flow described here is a flow of air at $10 \mathrm{~cm} / \mathrm{s}$ imposed at the entrance of the domain. Apart from the $Z=0$ boundary which is the plate itself, other boundaries are infinite tank-type boundaries, e.g. simulated quiescent air at 
ambient temperature. In the calculations the line defined by $X=0, Z=0$ is the leading edge of the flat plate. Therefore a singularity can be found at this location that behaves as the stagnation point of the plate. The flow is therefore deflected and a positive pressure perturbation develops at the very vicinity of this leading edge and leads to an Xwise pressure gradient thus to the acceleration of the fluid in that direction (Figure 2).

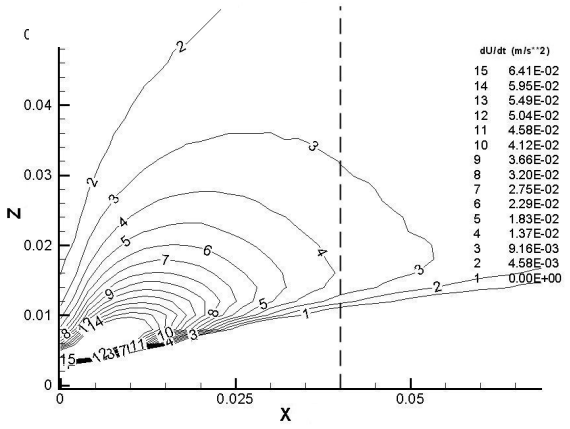

Figure $2 X$-wise acceleration distribution for a non-reactive flow. The fuel injection is set to zero and the free stream velocity is $0.1 \mathrm{~m} / \mathrm{s}$. The origin of the plate is set at $X=0$, $Z=0$ and the air flows for $Z>0$. The leading edge of the porous burner is placed at $X=0.04 m$ (dotted line)

The flow acceleration, manifested through the pressure increase close to the leading edge of the plate, mainly takes place close to the entrance of the domain and roughly extends up to the leading edge of the porous burner. If the boundary layer thickness where to be traced, the pressure increase occurs just above the boundary layer. This behaviour corresponds well with that described by Ha et al. [21]. It is important to point out that in these computations, due to the calculation set-up, the stagnation point is located exactly at the edge of the plate, as for an extremely thin flat plate. In the experiments described by $\mathrm{Ha}[21]$ a flat plate $(Z>0)$ with a leading edge making an angle of $45^{\circ}(Z<0)$ was been used to avoid flow separation at the leading edge of the plate. The effect of this inclined surface under the plate is to push the stagnation point below the $Z=0$ plane. This difference leads to a different position of the pressure perturbation and therefore to a different position of the acceleration zone. However, as shown, the strongest acceleration zone is mostly located in the vicinity of the entrance of the domain, e.g. for $\mathrm{X}<0.025$. Past this point, acceleration decreases and is no longer significant when the flow reaches the leading edge of the porous burner $(X=0.04)$. Figures 3 and 4 show the velocity components in the $\mathrm{X}$ direction scaled by $\mathrm{U}_{\infty}$ and in the $\mathrm{Z}$ direction scaled by a characteristic value for $\mathrm{W}_{\mathrm{f}}$. Throughout this presentation a characteristic value of $\mathrm{W}_{\mathrm{f}}=$ $3 \mathrm{~mm} / \mathrm{s}$ will be used as a reference to describe the different phenomena observed. These velocity profiles are shown for six different positions on the $\mathrm{X}$-axis and all correspond to the plane $y=Y_{\max } / 2$, which is the centre-plane of the domain. For convenience a new variable is defined, $\xi=\mathrm{x}+0.04 \mathrm{~m}$, and corresponds to a translation of the coordinate axis from the plate leading edge to the burner leading edge. Also the square burner characteristic length scale will be defined as the length of a side and denoted as $\mathrm{L}(\mathrm{L}=$ $60 \mathrm{~mm}$ ). Thus, $\xi=0$ corresponds to the leading edge of the porous burner, $\xi=\mathrm{L} / 2$ to the centre of the porous burner and $\xi=\mathrm{L}$ to the trailing edge of the porous burner. As can be seen from Figure 3, the plate accelerates the flow beyond the magnitude of the free stream velocity. The velocity profiles show an overshoot of about $11 \%$ in the X-direction 
followed by a very slow decay towards the nominal free stream velocity. This acceleration is slightly higher for $\xi=0$, since it is the closest point to the plate leading edge, but nevertheless can be consider an almost constant acceleration of the free stream. The weak decay in the $\mathrm{Z}$ direction further supports the assumption that the acceleration can be accounted by a correction. Figure 4 shows clearly that the pressure gradient induced by the plate leads to a vertical deflection of the flow. This deflection is not really significant since vertical velocities are always less than $5 \%$ of $\mathrm{U}_{\infty}$, approaching this value close to the leading edge of the porous burner and decreasing below $2 \%$ downstream. Small negative vertical velocities $\left(<1.6 \%\right.$ of $\left.\mathrm{U}_{\infty}\right)$ also appear at the top of the domain, revealing the shear boundary layer developed between the imposed flow and quiescent air at the boundary.

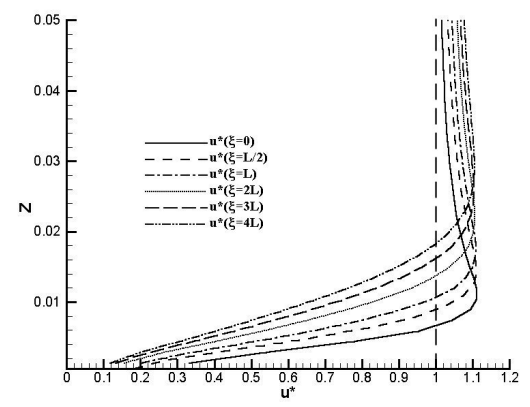

Figure 3 Normalized horizontal velocity, $u^{*}$ $\left(u^{*}=u / U_{\infty}\right)$, the fuel injection is $w_{f}=0 \mathrm{~mm} / \mathrm{s}$ and the flow is nonreactive.

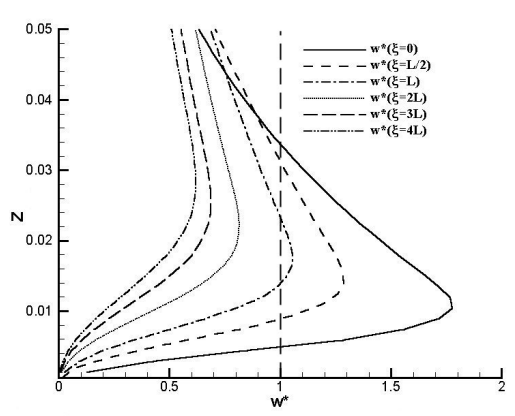

Figure 4 Normalized vertical velocity, $w^{*}$ $\left(w^{*}=w / W_{f}\right)$, the fuel injection is $w_{f}=0 \mathrm{~mm} / \mathrm{s}$ and the flow is nonreactive.

It is clear from these results that the simple presence of the plate does have a significant effect on the flow. Different plate geometries can be used to attempt better reproduction of a free stream boundary layer [21] but none of these geometries fully reproduces the idealized scenario. The plate thickness was varied and the same flow was modelled. This showed that the magnitude of the perturbations varies but the overall observations remain the same. Although this perturbations might seem important they have to be contrasted with those induced by the fuel injection and thermal gradients before reaching conclusions on how the plate might affect the assumption of an idealized free stream flow proposed by Emmons [10].

\section{Fuel Injection}

For the purpose of this study, air will be injected through the porous burner. The relevant experimental studies use ethane as fuel to best approach equal densities between fuel and oxidizer, therefore this simplification seems appropriate [22]. As mentioned before, the reference injection velocity that will be used to describe the different phenomena will be $\mathrm{W}_{\mathrm{f}}=3 \mathrm{~mm} / \mathrm{s}$. This value is of similar order to characteristic velocities induced by fuel pyrolysis and is also of similar magnitude to the vertical velocities generated by the plate. The X-wise acceleration distribution for a similar $U_{\infty}=0.1 \mathrm{~m} / \mathrm{s}$ case is presented in Figure 5. Close to the leading edge of the plate the positive pressure perturbation remains of the same magnitude and seems unaffected by the fuel injection. Nevertheless, the pressure increase decays much later than for the no injection case, 
therefore, acceleration of the flow will prevail almost through the entire plate. At the leading edge of the porous burner, a second zone of higher pressure is installed. This second perturbation is generated by an "obstacle" effect that the injection has on the main flow. These two combined pressure perturbations lead to a favourable pressure gradient developing from the entrance of the domain and extending above the porous burner. For this particular case, the zone of significant $\mathrm{x}$-wise acceleration now covers $65 \%$ of the porous burner as can be seen in Figure 5.

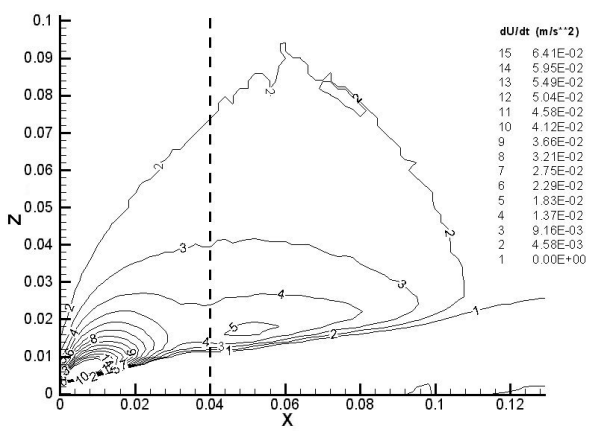

Figure $5 \quad X$-wise acceleration distribution for a non-reactive flow. The fuel injection is set to $W_{f}=3 \mathrm{~mm} / \mathrm{s}$ and the free stream velocity is $0.1 \mathrm{~m} / \mathrm{s}$. The origin of the plate is set at $X=0, Z=0$ and the air flows for $Z>0$. The leading edge of the porous burner is placed at $X=0.04 \mathrm{~m}$ (dotted line)

The corresponding velocity profiles are shown in figures 6 and 7. The maximum velocity overshoot is now located farther downstream in $\xi=\mathrm{L}$ and is around $11.4 \%$ of $\mathrm{U}_{\infty}$. The increase in velocity is not really significant, even if clearly visible, showing that injection does not lead to a dramatic acceleration of the flow. The maximum velocity at $\xi=0$ is slightly lower than in the case without injection, showing a further acceleration for $\xi>0$. This is a clear representation of what is referred here as the "obstacle" of injection to the free stream. Vertical velocity profiles show the significant deflection of the flow generated by the presence of injection (Figure 7). Nevertheless, the peak values for both components of the velocity seem to occur at the same location with and without injection, separation is not evident and the structure of the shear layer seems to remain fundamentally unaffected. Figure 7 also shows that there is a $60 \%$ increase in the magnitude of the vertical component to the velocity for $\xi=0$ with a further increase for $\xi=\mathrm{L} / 2$. The increase in vertical velocity remains present until the trailing edge of the burner, $\xi=\mathrm{L}$, is reached, beyond the trailing edge $(\xi=2 \mathrm{~L}, \xi=3 \mathrm{~L}$ and $\xi=4 \mathrm{~L})$ the vertical velocity remains almost the same than in the reference case. It is important to note that for $Z=0$, in $\xi=0$ and $\xi=\mathrm{L}$, the value of $\mathrm{w}^{*}$ is 0.5 and not 1 as expected. This is due to the fact that scalars are calculated at cell centres and velocities are calculated at cell edges. Therefore the obtained velocity, right at the centre of the first and the last cell of the burner, is the mean of the value for upstream and downstream faces of the cell. These velocity profiles show that injection acts as an obstacle that deflects the main flow and stretches the acceleration zone from the leading edge of the plate to the first third of the porous burner. Nevertheless, this "obstacle" introduced by injection does not result in a significant increase in thickness of the shear layer, nor a great acceleration of the free stream. 


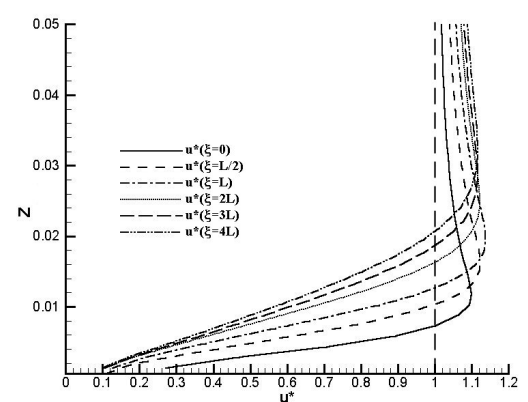

Figure 6 Normalized horizontal velocity, $u^{*}$ $\left(u^{*}=u / U_{\infty}\right)$, the fuel injection is $w_{f}=3 \mathrm{~mm} / \mathrm{s}$ and the flow is nonreactive.

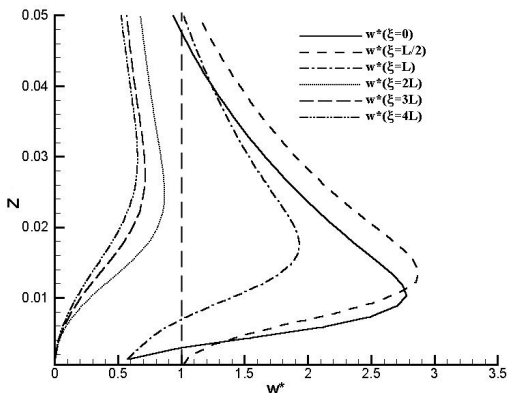

Figure 7 Normalized vertical velocity, $w^{*}$ $\left(w^{*}=w / W_{f}\right)$, the fuel injection is $w_{f}=3 \mathrm{~mm} / \mathrm{s}$ and the flow is nonreactive.

The Influence of Energy Release

The final stage of the calculations is to incorporate the combustion reaction. Fuel is now injected through the porous burner and combustion is simulated according to the mixture fraction model. The maximum velocity overshoot remains in $\xi=\mathrm{L}$ and is now around $12.3 \%$ of $U_{\infty}$. The maximum velocity at the leading edge of the burner $(\xi=0)$ is lower than for the cold flow. In contrast, at the same location the vertical velocity is negative close to the surface but reaches almost $10 \mathrm{w}_{\mathrm{f}}$ (which corresponds to $30 \%$ of $\mathrm{U}_{\infty}$ ) at its peak. Throughout the burner area the vertical velocity seems to become negative immediately above the burner and only reach a positive peak $30 \mathrm{~mm}$ above the burner. Down stream of the trailing edge the flow is dramatically distorted by the flame but negative vertical velocities cannot be observed anymore. It is of extreme importance to note that the peaks velocity values are attained for $Z>0.02 \mathrm{~m}$ which is double the distance at which these peak values were observed with a non-reactive flow. Figures 8 and 9 clearly point out the combined influence of thermal expansion and injection. Thermal expansion enhances the strength of the "obstacle," but without the "obstacle" $(\xi>\mathrm{L})$ the perturbations induced by thermal expansion will be greatly reduced.

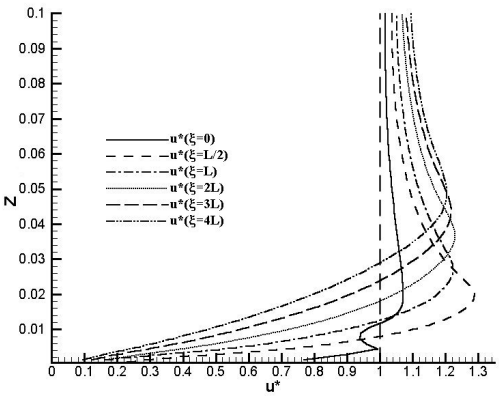

Figure 8 Normalized horizontal velocity, $u^{*}$ $\left(u^{*}=u / U_{\infty}\right)$, the fuel injection is $w_{f}=3 \mathrm{~mm} / \mathrm{s}$ and the flow is reactive.

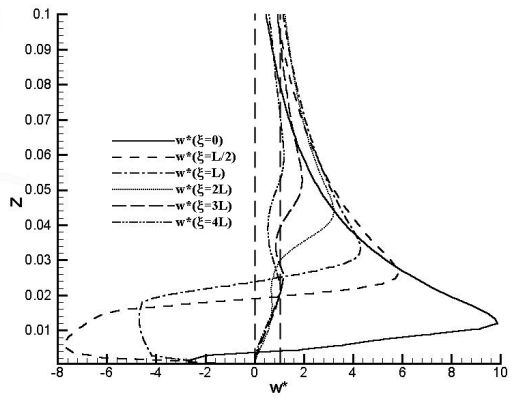

Figure 9 Normalized vertical velocity, $w^{*}$ $\left(w^{*}=w / W_{f}\right)$, the fuel injection is $w_{f}=3 \mathrm{~mm} / \mathrm{s}$ and the flow is reactive. 


\section{Validation}

Validation of this numerical approach has been done on the basis of comparison between the zone of maximum energy release and the boundary of the visible flame. Although this validation scheme is just qualitative it does provide a good indication of the accuracy with which the code describes the macroscopic variables of the process, such as the flow. Figure 10 shows, for the same airflow and injection regimes presented in this paper, experiments performed in micro-gravity. All the experiments were conducted on board of the AIRBUS A300 ZERO-G during a CNES Parabolic flight campaign. The experimental configuration corresponds to the calculation domain (physical dimensions, flow and injection velocities). Details of the hardware and experimental protocols can be found in reference [11]. Figure 10 is a CCD camera instantaneous shot of the flame. Figure 11 shows the calculated temperature field for the same experimental conditions. The grey dashed line represents heat release rate zone, that is to say the reaction zone. The comparison of these images serves as a validation tool for the present approach. As can be seen, the evolution of the stand-off distance observed in the images is in good agreement with the calculations. The highest luminous intensity gradient (transition from black to white in Figure 10) corresponds well with the maximum energy release zone (grey dotted lines in Figure 11). Comparisons were done for a number of different conditions and in all cases agreement seems to be comparable to the condition that has been used for illustration.

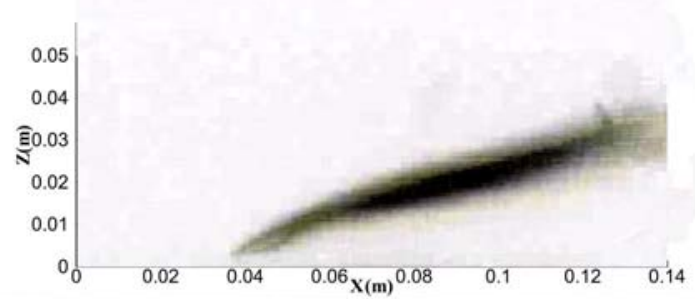

Figure 10 Micro-gravity experiments visible flame. The fuel injection is set to $W_{f}=3 \mathrm{~mm} / \mathrm{s}$ and the free stream velocity is $0.1 \mathrm{~m} / \mathrm{s}$.

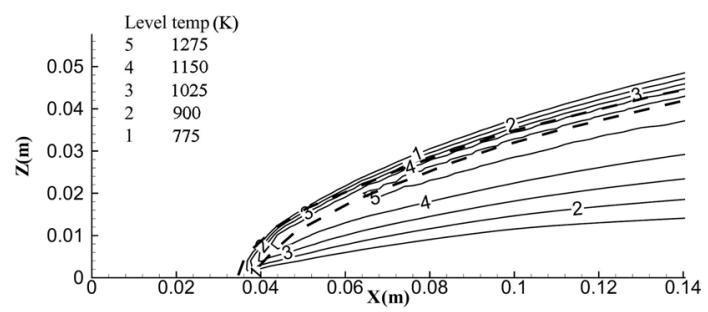

Figure 11 Temperature distribution for a reactive flow and the heat release zone (grey dashed contour). The fuel injection is set to $W_{f}=3 \mathrm{~mm} / \mathrm{s}$ and the free stream velocity is 0.1 $\mathrm{m} / \mathrm{s}$. The origin of the plate is set at $X=0, Z=0$ and the air flows for $Z>0$. The leading edge of the porous burner is placed at $X=0.04 \mathrm{~m}$

\section{CONCLUSION}

A numerical study on the flow characteristics of a diffusion flame in micro-gravity environment has been conducted. Flow velocities were established to simulate heat and 
ventilation systems on board of spacecraft. The fuel is simulated by gas injection through a porous burner. The fuel injection speeds are set to be comparable to pyrolysis. Numerous studies have concentrated on the flow perturbations induced by the solid fuel (in this case the burner) and on the influence of thermal expansion and fuel injection. Nevertheless, none of these studies has provided a clear quantification of the importance of these effects, especially in what relates to validation of the assumptions of classical analytical formulations. I was observed that pressure perturbations at the leading edge of the plate have to be seriously taken into account since it leads to non-negligible velocity overshoots and could help or cause flow separation. Nevertheless, if separation is prevented the ultimate effect of these perturbations on the flow downstream is minor. Fuel injection at velocities comparable to those induced by pyrolysis will create an obstacle for the flow, enhancing the effect of the pressure perturbations. But again, the effect is minor and tends to diffuse downstream of the plate. Separation does not seem to occur at these small injection velocities. Other simulations, using higher injection velocities, show that eventually separation will occur. Energy release seems to be the major source of flow acceleration for such a regime strongly influencing the boundary layer thickness. Strong temperature gradients close to the burner induce flows that could reverse the direction of the fuel. This study has provided an in-depth analysis that shows that the main parameter to consider when evaluating the precision of an analytical model should be the influence of the thermal expansion on the flow. Other parameters, such as geometry and injection, can be corrected to incorporate their minor effects into the analysis.

Acknowledgements

This work was funded by CNES and ESA in Europe. JLT was supported by NASA and the Minta Martin Research Foundation at the University of Maryland.

\section{REFERENCES}

[1] Sherman, F.S., “Viscous Flow,” McGraw-Hill, 1990.

[2] Friedman, R., "Fire Safety in Extraterrestrial Environments," NASA/TM-1998207417.

[3] Friedman, R., "Fire safety practices and Needs in Human-Crew Spacecraft," Journal of Applied Fire Safety, 2(3), 243-259, 1992-93.

[4] Friedman, R., "Fire Safety in Spacecraft," Fire and Materials, 20, 235-243, 1996.

[5] Ross, H. D., "Burning To Go: Combustion of Orbit and Mars," Fall Technical Meeting, The Eastern States Section of the Combustion Institute, 29-36, October 27-29, 1997.

[6] Torero, J.L., Bahr, N.J., Carman, E.J., "Assessment of Material Flammability for Micro-Gravity Environments" 48th International Astronautical Federation Congress, Turin, Italy, IAF-97-J.2.02, October 1997.

[7] T'ien, J.S. "The Possibility of a Reversal of Material Flammability Ranking from Normal Gravity to Microgravity," Combustion and Flame, 80, 355-357, 1990.

[8] Law, C.K. and Faeth, G. M. "Opportunities and Challenges of Combustion in Micro-Gravity" Progress in Energy and Combustion Science, 20, 1, 65-113, 1994.

[9] Ronney, P. D., "Understanding Combustion Processes Through Microgravity Research," Twenty-Seventh International Symposium on Combustion, Combustion Institute, Pittsburgh, 1998 (in press). 
[10] Emmons, H., "The Film Combustion of Liquid Fuel," Z.Angew. Math. Mech., 36: 60-71 (1956).

[11] T. Vietoris, P. Joulain and J. L. Torero "Experimental Observations on the Geometry and Stability of a Laminar Diffusion Flame in Micro-Gravity," Sixth International Symposium on Fire Safety Science, 373-386, 1999.

[12] G. Legros, K. Blase, J.L. Torero and P. Joulain, "Evaluation of a Realistic Mass Transfer Number from Images of an Upward Spreading Flame," $2^{\text {nd }}$ Joint Sections Meeting of the Combustion Institute, March 2001.

[13] Hirano, T., Iwai, K. and Kanno, "Measurement of the Velocity Distribution in the Boundary Layer over a Flat Plate with a Diffusion Flame," Y., Astronautica Acta, 17:811-818 (1972).

[14] Hirano, T. and Kanno, Y., "Aerodynamic and Thermal Structures of the Laminar Boundary Layer over a Flat Plate with a Diffusion Flame," Fourteenth Symposium (International) on Combustion, The Combustion Institute, Pittsburgh, 1973, pp.391-398.

[15] Ramachandra, A. and Raghunandan, B.N., "Investigations on the Stability and Extinction of a Laminar Diffusion Flame Over a Porous Flat Plate," Combustion Science and Technology, 36:109-121 (1984).

[16] Lavid, M. and Berlad, A.L., "Gravitational Effects on Chemically Reacting Boundary Layer Flows over a Horizontal Flat Plate," $16^{\text {th }}$ Symposium (International) on Combustion, The Combustion Institute, 1157-1568, 1976.

[17] Andreussi, P. and Petraca, L. "Film Combustion of Ethyl Alcohol in a Parallel Air Stream," Eighteenth Symposium (International) on Combustion, The Combustion Institute, Pittsburgh, 1981, pp.1861-1869.

[18] Andreussi, P. "Modelling of Laminar Diffusion Flames over a Horizontal Plate," Combustion and Flame, 45, 1-6 (1982).

[19] Andreoti, S., Andreussi, P. and Petraca, L. "Boundary Layer Burning of Fuel Surfaces: Thermal and Aerodynamic Structure of the Flame," Combustion Science and Technology, 40, 279-291 (1984).

[20] Nakagawa, Y., Nishiwaki, N. and Hirata, M. "Effect of Combustion on a Laminar Boundary Layer," Thirteenth Symposium (International) on Combustion, The Combustion Institute, 813-819, 1971.

[21] Ha, J.S., Shim, S.H. and Shin, H.D., "Boundary layer Diffusion Flame over a Flat plate in the Presence and Absence of Flow Separation," Combustion Science and Technology, 75, 241-260, 1991.

[22] Torero J.L., Bonneau L., Most J.M., Joulain P., "The Effect of Gravity on a Laminar Diffusion Flame Established over a Horizontal Flat Plate," 25th Symposium (International) on Combustion, The Combustion Institute, 17011709, 1994.

[23] Torero J.L., Wang H-Y., Joulain P. and Most J-M. "Flat Plate Diffusion Flames: Numerical Simulation and Experimental Validation for Different Gravity Levels", Lecture Notes in Physics, 464, 401-413, 1995.

[24] T. Vietoris, J. L. Ellzey, P.Joulain, S.N. Mehta and J.L. Torero, "Laminar Diffusion Flame in Micro-Gravity: The Results of the Mini-Texus 6 Sounding Rocket Experiment, Proceedings of the Combustion Institute, 28, 2000.

[25] McGrattan K.B., Baum H.R., Rehm R.G., Hamins A., Forney G.P., Floyd J.E. "Fire Dynamics Simulator - Technical Reference Guide (Version 2)"

[26] C. Huggett "Estimation of the rate of heat release by means of oxygen consumption measurments", Fire and Materials, 4:61-65, 1980 